\title{
The Michel Benoist and Robert Mulholland Yearly European Spine Journal Review: a survey of the "surgical and research" articles in the European Spine Journal, 2013
}

\author{
Robert C. Mulholland
}

Received: 29 November 2013/Published online: 3 January 2014

(C) Springer-Verlag Berlin Heidelberg 2013

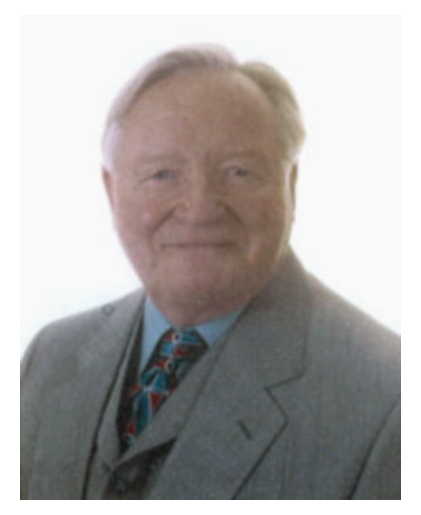

\section{Review articles}

There have been fewer review articles this year and although all of educational value, some were dealing with fairly arcane subjects. However, they perform a valuable educational role in providing a scientific basis for an opinion and are of value to anyone embarking on writing a paper in the field reviewed.

The paper on odontoid fractures [1] seeking to define the optimal treatment of odontoid fractures Type 11 and 111 as shown by a systemic review of the literature concludes that there is insufficient data as to whether surgical or conservative treatment is better, but do conclude that the majority

\section{Introduction}

Although the size of the journal has remained about the same (but with an extra supplement) the number of articles has increased from 273 to 323 . As was the case last year more have come from outside Europe, (152 from Europe, 184 from outside). China (62), Japan (36) and USA (28) are the major contributors but 17 other countries are represented. An important development is the number of combined studies between countries. It will be appreciated that those I have chosen to comment on are those, which my personal experience allows me to do so. I hope readers will look at the papers I have drawn their attention to and be tempted into reading other papers in the journal.

R. C. Mulholland ( $\square)$

34 Regent Street,

Nottingham NG1 5BT, UK

e-mail: MULHOLLANDRCM@aol.com of patients achieve fracture stability regardless of applied treatment. It does not alter my view expressed last year that the paper of Pal et al. [2] suggesting that treatment with a firm collar was in many cases the most appropriate treatment.

The review of the value of MRI in detecting posterior ligamentous injuries in patients with fractures in the thoracic and lumbar spine [3] came to the conclusion, rather to my surprise that there was scant clinical evidence demonstrating the true prognostic value of detected PLC injuries, and further longitudinal follow-up studies were required.

The review by Korse et al. [4] concerning the prognosis of bowel, bladder and sexual function after decompression for a cauda equina lesion, suffered as the length of followup of all the literature he explored was a mean of only 17 months. They suspected that further improvement would occur, as indeed the paper by Dhatt et al. [5] showed in his review some 2 years ago. They looked at the outcome of spinal decompression in cauda equina syndrome presenting late, in developing countries in 50 patients. The important point they make is that improvement occurs over a period of 2 or 3 years. 
The article "The evidence on surgical intervention for low back disorders-an overview of systemic reviews" illustrates the importance of reading the article rather than just the abstract. In the abstract it states that fusion rate favoured instrumented fusion over none instrumented, when one read the article, it was clear this did not mean a better clinical result. Perhaps the valuable aspect of the paper was it detailed all the problems of pooled information, and how the nature of the study created bias, i.e. what sort of patient is happy to be randomised?

The review by Smith et al. comparing open discectomy to micro-endoscopic discectomy concluded that both gave similar results, and had similar complications. I was a little surprised that one of their studies showed VAS was higher at $12 \mathrm{~h}$ in the micro endoscopic (MED) group, yet the authors concluded that this procedure could speed up recovery time. They also concluded that because of the longer learning curve for MED its use should be restricted to centres where the surgeons were experienced in endoscopic surgery.

\section{Back pain}

In 2001 Stirling [6-9] using serological tests demonstrated [10] that culture of intervertebral disc material from 36 patients operated upon for severe sciatica, showed that $53 \%$ grew Propionebacterium acnes. In the knowledge that Modic changes-bone oedema are present in only $6 \%$ of the general population [11], but are present in $35 \%$ of a back pain population, and the knowledge that these changes may be due to infection, Albert et al. [12] asked the question whether nuclear tissue so infected led to such changes. Disc material was cultured in 61 patients operated upon for a disc herniation, and these patients had MRI scans at the start, and at a mean of 424 days after surgery. Eight-six percent of those patients with a positive culture for an anaerobic organism, developed Modic changes, compared with $44 \%$ of those who had negative cultures, or cultures for an aerobic organism. This was highly significant and prompted the next paper [13] assessing the value of antibiotics in treating patient who had low back pain after a disc herniation (surgically treated or not) and who had Modic Type 1 changes. A very specific antibiotic protocol was devised; a prospective randomised, and blinded study with some 162 patients, 77 taking antibiotics, and 67 placebo pills. In the antibiotic treated group, there was considerable and significant improvement in pain experienced, time off work, and leg pain. The authors conclude that in this very specific group of chronic low back pain, antibiotic treatment is effective. As one might expect this startling development (reminiscent of the discovery that peptic ulcer was due to helicobacter infection has led to considerable discussion [14-16] and even two editorials [8]). The paper by O'Dowd and Casey [16] deserves careful reading, emphasising not only the important significance of these papers, but also the fact that these are a small group of patients, not representative of a large population of patients with chronic low back pain.

I suspect that in the coming year these papers will generate considerable discussion.

It is generally accepted that in patients with chronic low back pain there are structural changes in the brain affecting how they deal with pain. The paper by Ivo et al. [10] utilising sophisticated imaging device-voxel-based morphometric, demonstrated structural changes in those parts of the brain that are known to handle pain. It was of interest that psychometric measures in the patients, showing depression and anxiety caused no such changes. However, the authors report also that chronic pain associated changes can be altered with effective treatment of the cause, seen for example in patients who had such changes, due to hip pain, which were reversed following total hip replacement. It would seem that these changes are a response to the pain and not a cause. The situation may be that unless a procedure removes all the pain, then the existence of these brain changes continues to amplify the remaining pain.

The significance of HIZ in relation to back pain is still debated. Hebelka and Hansson [17] looked at 41 patients with low back pain, and subjected them to a standard recumbent MRI and an axially loaded MRI. They found that axially loaded MRI did not affect the detection of HIZ. In an associated study they showed that using pressurecontrolled discography, there was no correlation between pain on discography and the presence of an HIZ that is the presence of an HIZ was not an indication of a painful disc. They discount the paper by Lam et al. [18], which suggested that the presence of a HIZ was predictive of a painful disc, on the basis that the above study did not use pressure-controlled discography. No doubt the debate concerning the significance of the HIZ will continue.

The paper concerning disc degeneration in the spine producing hip pain by de Schepper et al. [19] deserves careful reading. It is an important subject as all too frequently major hip replacements are carried out, for mild OA of the hip, when in fact the pain is from the back. I believe this has become more frequent as specialised hip surgeons are often not aware of the relationship. However, to my knowledge, spinal surgeons have operated on the spine, when the source of pain was from the hip. The authors have concentrated on lumbar root irritation caused by degeneration in the upper lumbar spine, but rather ignored the more important cause, that of referred pain from the lower lumbar spine, especially at L4/5. Degenerative spondylolisthesis is associated with groin pain, and also with hip osteoarthritis. In fact their figures do show the association of hip pain with 
disc space narrowing at $4 / 5$, though the relationship is greater at the higher levels. However, it will be appreciated that narrowing at the $4 / 5$ level is much more frequent than at the upper levels, so clinically the association with the lower levels is more important.

There is much experimental evidence that afferents in the sympathetic system are important in transmitting pain, particularly in low back pain, the rami communicantes at L1 [20]. Sadly despite this evidence the paper by Rigaud et al. [21] "Is section of the sympathetic rami communicantes by laparoscopy in patients with refractory low back pain efficient" demonstrates that it is not, and indeed some patients were temporally made worse. One suspects that trying to deal with back pain by local nerve destruction is always in the long-term unlikely to succeed, and perhaps the paper by Ivo et al. [10] referred to above explains why.

Two papers from Japan, the first dealing with incidence of back pain in the general population and the second dealing with back pain in army recruits, and the radiological findings in this group are of interest. The first paper by Fujii and Matsudaira [22] was able by using an Internet survey, and shopping point rewards to look at a large number of people $(77,709)$ and reach these people with a fairly detailed questionnaire. Although their definition of chronic pain as being more than 3 months was rather short, the overall figures they acquired, much the same as those in Europe and North America, of a lifetime prevalence of $83 \%$ and, a 4-week prevalence of $36 \%$. Factors they identified as being associated with chronic low back pain was much as expected, compensation, occupational, and traffic injury, indeed giving a cause for the pain was more likely to be associated with chronicity. The malign effect of back surgery was noted; they were ten times more likely to have chronic pain, compared with compensation patients, 2.4 times more likely. I thought the paper was of value as the methodology using the Internet may have a wider application to other medical surveys. The paper on military recruits by Nemoto et al. [23], identified 84 recruits in 1990, who had no back pain and normal X-rays. They then reviewed them 20 years later using a questionnaire and X-rays. Half of the group after 20 years had LBP lasting more than 7 days in the current year. In this group vertebral osteophytes were more common. However, disc space narrowing in the back pain group did not reach statistical significance. They found no lifestyle affects, looking at weight, smoking, alcohol and sport.

We are all aware of the lack of relationship of the severity of a back injury and whether it leads to chronic low back pain. However, the beautifully written paper by Patel [24] attempts to put substance into this impression. He compares the long-term disability and function of patients after a major spinal fracture, requiring surgery, and compares them to a population attending a medicolegal clinic, with self-reported injury (query any injury) and seeking compensation. The authors draw the conclusion that the compensation group had minor injury, and get chronic pain, whereas the major spinal injury patients do not, and the conclusion is that a structural injury is not a factor in chronic back pain. Because of a lack of what he terms "Dose response". Superficially it was all very persuasive until one read the superb destruction of the paper by Kanna [25]. The concept of the study was a good one, but one suspects that the medicolegal group was selected because the authors were confident that this would prove what they believed. The custom the journal has of occasionally putting a rebuttal beside a contentious paper, is well illustrated her, and is of great value.

\section{Fractures}

The AO Spine thoracolumbar fracture classification has now been in use for nearly 20 years [26] and has increasingly become the principle classification used by surgeons. However, in that time there has been much change in imaging, so that it is appropriate that the classification should be revisited and updated. The paper by Reinhold et al. [27] does this, making some suggested revisions to take account of changes in imagine, particularly our ability to image soft tissue injuries. It deserves careful reading, and the editorial by Aebi [28] further clarifies the issues. In that October journal are a number of other interesting fracture reviews [29, 30]. The first by Xing et al. concludes that although the evidence is weak, early rather than late stabilization of thoracolumbar fractures reduced length of stay, intensive care occupancy, ventilator days, but it was not established that mortality was reduced. The second paper by $\mathrm{Xu}$ et al. provided further evidence that in treating burst fractures at the thoracolumbar level, the posterior approach was preferable insofar as shorter operative time, less blood loss, and lower costs compared with an anterior approach, with equally satisfactory clinical outcome, in terms of neurological recovery, and return to work.

The use of percutaneous transpedicular screw fixation in the treatment of Hangman's fracture (traumatic spondylolisthesis of the axis) described by Wu et al. [31] describes a useful technique. Although only ten patients are reported, and it is clear that all such fractures are suitable, modern imaging can allow this technique, and clearly there is much less morbidity.

\section{Disc and vertebral body infection}

The paper by Duarte and Vaccaro [32] is an excellent overall review of management of discitis and vertebral 
body infection. The authors draw attention to the fact that fever may not be present on presentation and diagnosis is often long delayed. CPR would seem better serological test, especially as its level is a better guide to response to treatment than the ESR. The development of an endocarditis in some $30 \%$ of patients was a surprise to me. They emphasise the importance of obtaining bacteriological confirmation by needle biopsy. Unfortunately since these investigations are now frequently carried out by Radiologists, and they use fine diameter needles, and achieve only $70 \%$ positive culture, rather than using a $2 \mathrm{~mm}$ or more diameter needle, where positive bacteriology is obtained in over $90 \%$ of biopsies [7]. They draw attention to the indications for surgery, as intractable pain, instability, and neurological changes, but make the point that minor neurology may resolve. They emphasise the importance of bracing. In discussing surgical treatment I was surprised they felt pedicle fixation should be added to an adequate anterior clearance and grafting, even in the absence of any posterior destruction. The paper by Valancius et al. [33] reinforces much of the above. Of the 196 patients they treated with spondylodiscitis, 91 were treated conservatively, and 105 operatively. Again I was surprised at the fact that 76 were treated with a posterior approach, debriding and clearing the disc space, and fixing with pedicle screws and an intertransverse fusion. This was because the authors felt that anterior surgery alone with grafting was not sufficiently stable, and operating from both the front and back with separate approaches was too invasive. Their results seem to support the views that a posterior approach alone is satisfactory, using a transforaminal lumbar interbody fusion to clear the disc, and do an interbody graft, then combined with pedicle fixation [33]. The paper by Gorensek et al. [9] operated upon 23 of 108 patients, indicating that nonoperative treatment is most frequently most appropriate. Because neurological indications were frequent, in their series, decompression was necessary, and they achieved excellent results using a posterior approach alone, and the implantation of cages despite the presence of infection was not attended with failure.

The paper dealing with cervical pyogenic infections by Urrutia et al. [34] suggests that these are a more serious problem than vertebral infection in other parts of the spine. They found that of 102 spinal infections, 18 were in the cervical spine, and in this group the disease was associated with bone destruction, epidural abscess and 13 of the 18 had serious neurological changes. They reported an operative rate of $84 \%$, and a mortality of $21 \%$, the latter in part due to comorbidity, although one death was due to bacterial endocarditis. Their view is that early diagnosis, and prompt treatment, usually surgical was mandatory in this group of patients.
The concept that serious posterior disease could be treated by posterior approaches only was demonstrated in the paper by Zhang et al. [35] in treating tuberculosis. In fact at the diseased level they did a costo-transversectomy to do an adequate debridement, and then do an interbody fusion. As they did not need to collapse a lung, they had fewer post-operative pulmonary disturbances. The paper by Kumar et al. [36], also treating tuberculosis, does the same, but dispenses with any grafting, merely draining any abscess. The message is that chemotherapy and appropriate rigid fixation is effective is creating fusion and correction.

\section{Spinal stenosis}

Because we are as uncertain as to the cause of back pain, it is often the case that when doing a decompression for radicular symptoms in patients who have back pain, fusion is advocated as well, although in fact decompression alone most frequently cures back ache associated with spinal stenosis, if this is not associated with spondylolisthesis. The paper by Tsutsui et al. [37] addresses the issue whether it relieves the back pain when stenosis is associated with scoliosis. They found that the degree of apical rotation correlated with the likelihood of their having continued back pain. Apical rotation produces a lateral spondylolisthesis, and it is likely that this mechanical factor is the cause of the pain. Patients therefore with low or no apical rotation associated with their scoliosis, need only decompression.

The debate about the efficacy of interspinous spacers in treating spinal stenosis, especially in the long-term continues. The paper by Beyer et al. [38] in which the authors compared the results at 2 years, of 12 patients who had had a spacer inserted, with 33 who had an open decompression. Walking distance improved in both groups, but of the 12 patients treated with a spacer, 5 had the implant removed and had an open decompression, and the remaining 6 reported worse results as compared with the remaining 26 patients from the open decompression group ( 7 lost to follow-up). Clearly this is a small non-randomised study but the review of the literature that is in the paper does not support the continued use of these implants for spinal stenosis.

Radicular pain in patients with spinal stenosis is commonly thought to be related to ischemia, so the use of vaso dilating medication would seem logical. Limaprost-a prostaglandin - has vasodilating properties and increases blood flow in nerves. The study by Beyer et al. [38] was a prospective randomised trial comparing its value with NSAID's. It had little effect on back pain, and indeed the best results were achieved if it was combined with NSAID's, as the latter were less effective for leg pain. 
Whether this therapy will gain acceptance will I suspect depend on the cost of the Limaprost, but this study would support its use.

\section{Kyphoplasty and vertebroplasty}

These procedures are gaining greater acceptance and the paper by Röder et al. [39] drawing attention to the importance of cement volume in achieving pain relief explains failures of some studies to confirm efficacy of the procedure. The combination of kyphoplasty with percutaneous pedicle fixation described by $\mathrm{He}$ et al. [40] is very persuasive. This was a prospective randomised trial comparing the two treatments. The patients who had kyphoplasty combined with percutaneous pedicle fixation had better pain relief, and better deformity correction, when reviewed at 2 years. Pedicle fixation was achieved through four small incisions and prebent rods were introduced through the paraspinal muscles. One advantage was that the correction of the deformity by the pedicle fixation produced a very obvious defect in the vertebra, so that the cement filled it, and leakage was not a problem. A paper earlier in the year dealt with the accuracy of percutaneous screwing, [41] found that in a prospective clinical trial using biplanar image intensification 494 out of 502 screws inserted were good or excellent. Only one screw out of the 502 inserted into 111 patients, had to be revised due to a neurological injury.

The value of vertebroplasty combined with posterior surgery is clearly shown in the paper by Kashii et al. [42]. They looked at 88 patients with osteoporotic vertebral collapse, with neurological deficits, and compared three procedures, anterior with neural decompression, posterior surgery, which consisted of a shortening osteotomy with direct neural decompression, and posterior fixation, with no direct decompression but combined with vertebroplasty. All three procedures were equally successful, but clearly the vertebroplasty was much less invasive. Direct neural decompression using this technique is not necessary.

\section{Tumours}

The scoring system described by Tokuhashi in 1990 to guide surgeons in making a decision as to whether surgical intervention is appropriate in treating a patient with spinal metastasis is well established. The paper by Gakhar et al. [43], which showed that in a retrospective review of some 90 patients, the survival rate was much better in patients who were expected to have a short survival rate as indicated by the score. Indeed $66.6 \%$ of their patients had a different actual survival rate from that predicted by the
Tokuhashi score. The authors do not comment on this, but as many of their patients were breast, myeloma, lymphoma, one suspect that now 13 years after the score was first described, the medical management of these tumours is much improved.

Another paper dealing with surgical treatment of spinal metastasis was that of Quraishi et al. [44]. They retrospectively reviewed 121 patients admitted and operated upon in their unit, 45 within 24 h, 23 between 24 and 48 h, and 53 after $48 \mathrm{~h}$. Although the time of surgery did not influence time of hospital stay, incidence of infection postoperative complications or survival, it did influence the degree of neurological recovery. Operation within $24 \mathrm{~h}$ was somewhat better than between 24 and 48, although not significant, but operation after $48 \mathrm{~h}$ was associated with poor neurological recovery.

\section{Disc replacement and fusion}

The paper by Skold et al. [45] is intriguing. At 2 years, the results of both procedures were much the same, whereas now at 5 years, disc replacement is better. There is what I take to be a misprint, when they state that 29 patients elected to have their prosthesis changed to a fusion, in an associated table, the figure is 9 . It is of interest that this group did less well in any event, and the authors conclude that such revisions are probably not indicated unless there is a clear failure of the implant. Why fewer of the TDR group were working at 5 years is not understood, as in the TDR group $59 \%$ were totally pain free, compared with only $38 \%$ in the fusion group. Two other papers adjacent to this dealt with the likelihood, for biomechanical reasons of TDR failure at the lumbosacral level [46, 47]. In doing a TDR at the lumbosacral level with marked narrowing of the disc, one must avoid increasing segmental lordosis, and posterior translation of the superior vertebrae. This produces tension or distraction of the facet joints, and in their clinical study, these features were associated with a less good result. Their paper would support the anecdotal view that at the lumbosacral level fusion may be the preferable option to TDR. It certainly supports the view that in gaining experience in doing a TDR the surgeon should start at a level above, unfortunately access wise more difficult.

Despite the fact that the results of fusion appear to be independent of the technique, there are still a bewildering array of techniques in use. However, the paper by Duncan et al. [48] shows that unilateral fixation by pedicle screws when doing a foraminal cage fusion is associated with cage migration. Minimally invasive techniques combined with TLIF naturally would wish to keep intervention to one side only, but this paper suggests that this would carry risk of cage migration. 
In the early part of the last century, sacroiliac fusion was in vogue for the treatment of back pain. However, its use faded throughout the century, but is now once again becoming fashionable in part because modern minimally invasive techniques make the procedure easier. The paper by Kibsgard et al. [49] is hence of great interest. They report on the results of patients who had sacroiliac fusion with a 25-year follow-up. Their conclusion was that at 25 years the patients had moderate disability and pain. Many fused patients reported good outcome, this group did not differ from a comparable non-surgical group. One indication that is in vogue now is to ascribe failure after lumbar fusion to pain arising from the sacroiliac joint, justifying fusion of that joint. However, the paper by Slinkard et al. [50] addresses this issue. They reviewed 19 patients who had had an anterior sacroiliac fusion, an open procedure, with anterior plating and grafting of the joint-a rather invasive procedure compared with the various image guided techniques. Of the 19, 12 had had a prior spinal fusion, and the results in this group were significantly inferior to the results in the 7 who had not had a prior spinal fusion. However, patients who had complications had less improvement.

All surgeons involved in doing fusions or decompressions are aware of the unpredictability of results, and have recognised the role that psychology plays in affecting outcome. The paper by Havakeshian and Mannion [51] clarifies the issues involved. They found that in a multivariable prospective mode; only a questionnaire dealing with Fear Avoidance Beliefs about physical activity (FABQ-PA) was the only base line psychological factor that significantly predicted outcome. Other psychological disturbances-depression, anxiety, and degree of disability-did not predict outcome, they improved post-operatively in patients with a good outcome and worsened in those with a bad outcome. They suggest that "prehabilitation" in which patients would have educational and psychological treatments to address maladaptive issues in those patients who have high levels preoperatively.

Should we do lumbar fusions in elderly patients? The paper by Anderson et al. [52] gives a rather ambiguous answer. They looked at long-term health care utilization after spinal fusion in elderly patients (between 59 and 80) and found that although their use of hospital care was much reduced, they made greater demands in primary care, than the general population in that age group. Although the reasons for this are not clear, the authors suggest that their increased fitness means that other musculo-skeletal problems, which they are likely to have, are treated in primary care.

\section{Spondylolisthesis}

An important development in recent years in surgery of the spine is our appreciation of the importance of sagittal balance. The paper by Harroud et al. [53] looked at 149 unoperated children with lumbosacral spondylolisthesis (117 low-grade and 32 high-grade) and found that in the high-grade group where there was a loss of normal sagittal balance, health related quality of life was significantly reduced, whereas this was not the case in low-grade slips. Low-grade slips had a much less effect of overall sagittal balance, as children and adolescents had sufficient mobility to compensate. This paper justifies therefore certainly reducing the kyphotic element in a slip and if possible reducing a high-grade slip, but does not support the need to reduce a low-grade slip.

\section{Cervical spine}

The systemic review and meta-analysis of the results of cervical decompression for spondylotic myelopathy when done anteriorly or posteriorly by Zhu et al. [54] although based on just eight studies, none of them prospective or randomised, came out with a clear message, multi-level decompression should be done from behind. Five hundred and thirty patients were studied, 245 patients had anterior surgery, and 285 posterior surgery. It is of interest that of those operated upon posteriorly only 83 had posterior fixation, 202 having a laminoplasty. One wonders if the low level of posterior fixation is related to the frequency of OPLL in the patients, as five of the eight studies were from Japan [4] and China [1]. Those not internally fixed had laminoplasties. Late development of kyphosis in the posteriorly operated group is not alluded to indicating the stability of laminoplasty. There were many fewer complications in those operated from behind, with equally good neurological recovery.

There were two interesting papers dealing with the locomotor defect in the myelopathic patient. The first from Japan [55] dealt with the results from decompression. They showed that taking account of the fact that there may be other neurological disorders in the older patient affect gait, surgery was well worth while, and should be done early. The second paper from Ireland examined the electromyographic characteristics of gait impairment in these patients [56]. This showed that although spasticity was always present, it was not a major factor in the gait problem, but weakness was. The authors rather coyly suggest that this has implications in assessment and rehabilitation of these patients, but it would clearly suggest that physiotherapy designed to strengthen muscles was most appropriate.

There is increasing concern about the effects of radiation now that various MISS procedures are increasingly used, so the paper by Obernauer et al. [57] reporting a prospective randomised trial comparing the use of ultrasound with CT guided periradicular injections in the middle and lower 
cervical spine is of some interest. The needle was guided by ultrasound, and the final position verified by $\mathrm{CT}$ in one group, and in the other CT was used throughout the procedure. The results with ultrasound-guided injections were as good and significant reduction in radiation was achieved.

Another paper which reduces radiation is that by McCracken et al. [58] dealing with the value of flexion and extension lateral radiographs to detect instability in awake patients, with neck pain, when using $\mathrm{CT}$ there was no apparent injury to the neck. They retrospectively reviewed 1,000 patients, and found that adequate flexion extension films were difficult to obtain and minimally helpful in clearing the cervical spine in awake and alert trauma patients. Such patients are being assessed because of neck pain, and the associated spasm prevents an adequate examination. They advocated a straight lateral film should be taken in such patients, and if there was a kyphosis, then an MRI scan should be done. Their chart of the current guidance on clearing the spine is excellent and could be usefully on display in an accident department.

It is always refreshing to be taught a new clinical test, and the arm squeeze test used to distinguish between pain in the shoulder area caused by a shoulder problem, and one due to cervical root irritation described by Gumina et al. [59] is a useful addition to our clinical skills. The clinical basis is that irritated nerves are tender (seen in the lower limb-as a positive bowstring test in sciatica due to an HNP) so in the upper limb, squeezing the upper arm one squeezes nerves from $\mathrm{C} 5$ to Ti. What is impressive about the paper is the size of the study (1,567 patients) and 350 controls. In patients with proven root irritation it was positive in $96.7 \%$ but only positive in 35 of 903 patients with a shoulder problem, and only positive in $4 \%$ in asymptomatic volunteers, a sensitivity of 0.96 and a specificity of $0.91-1$.

Dysphagia is a troublesome complication after anterior cervical surgery, and the paper by Fengbin et al. [60] suggests that the incidence can be reduced, if the anterior approach is varied slightly. They did a prospective randomised study involving 80 patients comparing the incidence of this complication in those where the approach was medial to the omohyoid muscle, and those in which it was lateral. They found that if surgery was to the levels 3-4, then it was preferable to go lateral to the omohyoid, if the surgery was at levels C6 or C7 then the surgeon should go medial to the omohyoid, and from the left side. The authors have useful discussion as to the cause of the dysphagia, and its probable relation to traction injury of the superior laryngeal nerve.

One of the reasons for the increasing use of disc replacement in the cervical spine is the view that it makes adjacent level degeneration less likely compared with a fusion. The paper by Park et al. [61] rather disposes of this myth. They compared 22 patients who had had an arthroplasty with 21 patients who had had fusion. Plain films, CT and MRI were done before surgery and at 5 years. The ASD rate was $46.5 \%$ and was the same in both groups. The authors conclude that ASD is related to the natural history of cervical spondylosis rather than arthrodesis.

\section{Supplements}

The concept of publishing supplements is of great educational value, at present there are national supplements, which include podium and poster presentations from the annual meeting of the national spine societies, focussed supplements, and a supplement containing papers and posters from the Eurospine annual meeting.

The national supplements show the great activity in our speciality in each country and ensure that presented papers are published and hence accessible. The focussed supplements are of considerable value as they bring the reader up to date with a particular field. This year the supplement on tuberculosis was most valuable, dealing with a disease that we realize is now on the increase. In this supplement there were some 15 papers, and an editorial. The supplement could be read through in an afternoon, and one was brought up to date on the overall challenge of the disease and how we were coping with it. Indeed the supplement is a succinct textbook dealing with Tuberculosis. I particularly enjoyed the paper by Millet et al. [62] dealing with factors that influence current tuberculosis epidemiology. The high incidence of drug resistance in Eastern Europe and the Russian Federation is alarming. The influence of smoking, especially when combined with poverty should provide further ammunition to reduce tobacco consumption. The paper by Rajasekaran and Khandelwal [63] was short, concise, and outlined very clearly the basis for the use of the various drugs, and emphasised that with early diagnosis tuberculosis is a medical disease. In 1978 Hong Kong was one of the centres that the Medical Research Council in the UK chose to assess the value of medical treatment and the role of surgery [64], and a number of spinal surgeons in the UK went to Hong Kong to be trained in anterior approaches to the spine. I found therefore the paper by Cheung and Luk [65] of great interest. What seems clear is that fusion and correction of deformity early in the disease is necessary to prevent later recurrence of the disease and increasing deformity, and this can be after 15 years-the follow-up time that the MRC chose. The difficulties and hazards of surgery to deal with severe late symptomatic post-TB kyphosis are such that even with the use of the technique described by Rajasekaran et al. [66], that primary treatment should achieve fusion and correct deformity. The review article in this supplement dealing with treatment of 
spinal tuberculosis with neurological defects by Jain and Kumar [67] gave an excellent overview of the clinical presentation, the surgical treatment, and the various surgical approaches.

Supplement 6, which included some 29 papers selected from the meeting in 2012 of the Italian Spine Society, had a number of interesting papers. The paper by Crostelli et al. [68] gives further evidence that posterior correction of severe adolescent scoliosis by the posterior approach only, but using pedicle screws throughout the thoracic spine is as effective, and in some respects better than combined anterior and posterior procedures, and with less morbidity.

In doing this review space has not allowed me to cover the Grand Rounds presentations, or the Open Operating Theatre, both very valuable educational developments in the journal. The latter provided the continued surgical education we now recognize as so important as spinal surgery develops.

Conflict of interest None.

\section{References}

1. Thomsen K, Christensen FB, Eiskjaer SP, Hansen ES, Fruensgaard S, Bunger CE (1997) 1997 Volvo Award winner in clinical studies. The effect of pedicle screw instrumentation on functional outcome and fusion rates in posterolateral lumbar spinal fusion: a prospective, randomized clinical study. Spine 22(24):2813-2822

2. Pal D, Sell P, Grevitt M (2011) Type II odontoid fractures in the elderly: an evidence-based narrative review of management. Eur Spine J Off Publ Eur Spine Soc Eur Spinal Deform Soc Eur Sect Cerv Spine Res Soc 20(2):195-204

3. van Middendorp JJ, Patel AA, Schuetz M, Joaquim AF (2013) The precision, accuracy and validity of detecting posterior ligamentous complex injuries of the thoracic and lumbar spine: a critical appraisal of the literature. Eur Spine J Off Publ Eur Spine Soc Eur Spinal Deform Soc Eur Sect Cerv Spine Res Soc 22(3):461-474

4. Korse NS, Jacobs WC, Elzevier HW, Vleggeert-Lankamp CL (2013) Complaints of micturition, defecation and sexual function in cauda equina syndrome due to lumbar disk herniation: a systematic review. Eur Spine J Off Publ Eur Spine Soc Eur Spinal Deform Soc Eur Sect Cerv Spine Res Soc 22(5):1019-1029

5. Dhatt S, Tahasildar N, Tripathy SK, Bahadur R, Dhillon M (2011) Outcome of spinal decompression in Cauda Equina syndrome presenting late in developing countries: case series of 50 cases. Eur Spine J Off Publ Eur Spine Soc Eur Spinal Deform Soc Eur Sect Cerv Spine Res Soc 20(12):2235-2239

6. Stirling A, Worthington T, Rafiq M, Lambert PA, Elliott TS (2001) Association between sciatica and Propionibacterium acnes. Lancet 357(9273):2024-2025

7. Fyfe IS, Henry AP, Mulholland RC (1983) Closed vertebral biopsy. J Bone Jt Surg Br Vol 65(2):140-143

8. Aebi M (2013) Is low back pain after disc herniation with Modic Type 1 changes a low-grade infection? Eur Spine J Off Publ Eur Spine Soc Eur Spinal Deform Soc Eur Sect Cerv Spine Res Soc 22(4):689

9. Gorensek M, Kosak R, Travnik L, Vengust R (2013) Posterior instrumentation, anterior column reconstruction with single posterior approach for treatment of pyogenic osteomyelitis of thoracic and lumbar spine. Eur Spine J Off Publ Eur Spine Soc Eur Spinal Deform Soc Eur Sect Cerv Spine Res Soc 22(3):633-641

10. Ivo R, Nicklas A, Dargel J, Sobottke R, Delank KS, Eysel P et al (2013) Brain structural and psychometric alterations in chronic low back pain. Eur Spine J Off Publ Eur Spine Soc Eur Spinal Deform Soc Eur Sect Cerv Spine Res Soc 22(9):1958-1964

11. Jensen TS, Karppinen J, Sorensen JS, Niinimaki J, Leboeuf-Yde C (2008) Vertebral endplate signal changes (Modic change): a systematic literature review of prevalence and association with non-specific low back pain. Eur Spine J Off Publ Eur Spine Soc Eur Spinal Deform Soc Eur Sect Cerv Spine Res Soc 17(11):1407-1422

12. Albert HB, Lambert P, Rollason J, Sorensen JS, Worthington T, Pedersen MB et al (2013) Does nuclear tissue infected with bacteria following disc herniations lead to Modic changes in the adjacent vertebrae? Eur Spine J Off Publ Eur Spine Soc Eur Spinal Deform Soc Eur Sect Cerv Spine Res Soc 22(4):690-696

13. Albert HB, Sorensen JS, Christensen BS, Manniche C (2013) Antibiotic treatment in patients with chronic low back pain and vertebral bone edema (Modic type 1 changes): a double-blind randomized clinical controlled trial of efficacy. Eur Spine J Off Publ Eur Spine Soc Eur Spinal Deform Soc Eur Sect Cerv Spine Res Soc 22(4):697-707

14. Ahmad Z, Rai A, Donell S, Crawford R (2013) Letter to the editor concerning: "Antibiotic treatment in patients with chronic low back pain and vertebral bone edema (Modic type 1 changes): a double-blind randomized controlled trial of efficacy" by Albert HB et al. Eur Spine J (2013) 22:697-707. Eur Spine J Off Publ Eur Spine Soc Eur Spinal Deform Soc Eur Sect Cerv Spine Res Soc 22(10):2344-2345

15. Albert HB (2013) Answer to the Letter to the Editor of K. Shubhakaran et al. entitled "Backache and infection" concerning "Antibiotic treatment in patients with chronic low back pain and vertebral bone edema (Modic type 1 changes): a double-blind randomized controlled trial of efficacy" by Albert HB et al. Eur Spine J (2013) 22:697-707. Eur Spine J Off Publ Eur Spine Soc Eur Spinal Deform Soc Eur Sect Cerv Spine Res Soc 22(10):2349

16. O'Dowd J, Casey A (2013) Antibiotics a cure for back pain, a false dawn or a new era? Eur Spine J Off Publ Eur Spine Soc Eur Spinal Deform Soc Eur Sect Cerv Spine Res Soc 22(8):1694-1697

17. Hebelka H, Hansson T (2013) HIZ's relation to axial load and low back pain: investigated with axial loaded MRI and pressure controlled discography. Eur Spine J Off Publ Eur Spine Soc Eur Spinal Deform Soc Eur Sect Cerv Spine Res Soc 22(4):734-739

18. Lam KS, Carlin D, Mulholland RC (2000) Lumbar disc highintensity zone: the value and significance of provocative discography in the determination of the discogenic pain source. Eur Spine J Off Publ Eur Spine Soc Eur Spinal Deform Soc Eur Sect Cerv Spine Res Soc 9(1):36-41

19. de Schepper EI, Damen J, Bos PK, Hofman A, Koes BW, Bierma-Zeinstra SM (2013) Disk degeneration of the upper lumbar disks is associated with hip pain. Eur Spine J Off Publ Eur Spine Soc Eur Spinal Deform Soc Eur Sect Cerv Spine Res Soc 22(4):721-726

20. Suseki K, Takahashi Y, Takahashi K, Chiba T, Yamagata M, Moriya H (1998) Sensory nerve fibres from lumbar intervertebral discs pass through rami communicantes. A possible pathway for discogenic low back pain. J Bone Jt Surg Br Vol 80(4):737-742

21. Rigaud J, Riant T, Labat JJ, Guerineau M, Robert R (2013) Is section of the sympathetic rami communicantes by laparoscopy in patients with refractory low back pain efficient? Eur Spine J Off Publ Eur Spine Soc Eur Spinal Deform Soc Eur Sect Cerv Spine Res Soc 22(4):775-781 
22. Fujii T, Matsudaira K (2013) Prevalence of low back pain and factors associated with chronic disabling back pain in Japan. Eur Spine J Off Publ Eur Spine Soc Eur Spinal Deform Soc Eur Sect Cerv Spine Res Soc 22(2):432-438

23. Nemoto O, Kitada A, Naitou S, Tsuda Y, Matsukawa K, Ukegawa Y (2013) A longitudinal study for incidence of low back pain and radiological changes of lumbar spine in asymptomatic Japanese military young adults. Eur Spine J Off Publ Eur Spine Soc Eur Spinal Deform Soc Eur Sect Cerv Spine Res Soc 22(2):453-458

24. Patel MS, Sell P (2013) Dose response and structural injury in the disability of spinal injury. Eur Spine J Off Publ Eur Spine Soc Eur Spinal Deform Soc Eur Sect Cerv Spine Res Soc 22(3):515-520

25. Kanna RM (2013) Reviewer's comment concerning "doseresponse and structural injury in the disability of spinal injury" (doi:10.1007/s00586-012-2498-2 by M. S. Patel et al.). Eur Spine J Off Publ Eur Spine Soc Eur Spinal Deform Soc Eur Sect Cerv Spine Res Soc 22(3):521-522

26. Magerl F, Aebi M, Gertzbein SD, Harms J, Nazarian S (1994) A comprehensive classification of thoracic and lumbar injuries. Eur Spine J Off Publ Eur Spine Soc Eur Spinal Deform Soc Eur Sect Cerv Spine Res Soc 3(4):184-201

27. Reinhold M, Audige L, Schnake KJ, Bellabarba C, Dai LY, Oner FC (2013) AO spine injury classification system: a revision proposal for the thoracic and lumbar spine. Eur Spine J Off Publ Eur Spine Soc Eur Spinal Deform Soc Eur Sect Cerv Spine Res Soc 22(10):2184-2201

28. Aebi M (2013) AO spine classification system for thoracolumbar fractures. Eur Spine J Off Publ Eur Spine Soc Eur Spinal Deform Soc Eur Sect Cerv Spine Res Soc 22(10):2147-2148

29. Xing D, Chen Y, Ma JX, Song DH, Wang J, Yang Y et al (2013) A methodological systematic review of early versus late stabilization of thoracolumbar spine fractures. Eur Spine J Off Publ Eur Spine Soc Eur Spinal Deform Soc Eur Sect Cerv Spine Res Soc 22(10):2157-2166

30. Xu GJ, Li ZJ, Ma JX, Zhang T, Fu X, Ma XL (2013) Anterior versus posterior approach for treatment of thoracolumbar burst fractures: a meta-analysis. Eur Spine J Off Publ Eur Spine Soc Eur Spinal Deform Soc Eur Sect Cerv Spine Res Soc 22(10):2176-2183

31. Wu YS, Lin Y, Zhang XL, Tian NF, Sun LJ, Xu HZ et al (2013) Management of hangman's fracture with percutaneous transpedicular screw fixation. Eur Spine J Off Publ Eur Spine Soc Eur Spinal Deform Soc Eur Sect Cerv Spine Res Soc 22(1):79-86

32. Duarte RM, Vaccaro AR (2013) Spinal infection: state of the art and management algorithm. Eur Spine J Off Publ Eur Spine Soc Eur Spinal Deform Soc Eur Sect Cerv Spine Res Soc 22:2787-2799

33. Valancius K, Hansen ES, Hoy K, Helmig P, Niedermann B, Bunger C (2013) Failure modes in conservative and surgical management of infectious spondylodiscitis. Eur Spine J Off Publ Eur Spine Soc Eur Spinal Deform Soc Eur Sect Cerv Spine Res Soc 22(8):1837-1844

34. Urrutia J, Zamora T, Campos M (2013) Cervical pyogenic spinal infections: are they more severe diseases than infections in other vertebral locations? Eur Spine J Off Publ Eur Spine Soc Eur Spinal Deform Soc Eur Sect Cerv Spine Res Soc 22:2815-2820

35. Zhang H, Sheng B, Tang M, Guo C, Liu S, Huang S et al (2013) One-stage surgical treatment for upper thoracic spinal tuberculosis by internal fixation, debridement, and combined interbody and posterior fusion via posterior-only approach. Eur Spine J Off Publ Eur Spine Soc Eur Spinal Deform Soc Eur Sect Cerv Spine Res Soc 22(3):616-623

36. Kumar MN, Joseph B, Manur R (2013) Isolated posterior instrumentation for selected cases of thoraco-lumbar spinal tuberculosis without anterior instrumentation and without anterior or posterior bone grafting. Eur Spine J Off Publ Eur Spine Soc Eur Spinal Deform Soc Eur Sect Cerv Spine Res Soc 22(3):624-632

37. Tsutsui S, Kagotani R, Yamada H, Hashizume H, Minamide A, Nakagawa Y et al (2013) Can decompression surgery relieve low back pain in patients with lumbar spinal stenosis combined with degenerative lumbar scoliosis? Eur Spine J Off Publ Eur Spine Soc Eur Spinal Deform Soc Eur Sect Cerv Spine Res Soc 22(9):2010-2014

38. Beyer F, Yagdiran A, Neu P, Kaulhausen T, Eysel P, Sobottke R (2013) Percutaneous interspinous spacer versus open decompression: a 2-year follow-up of clinical outcome and quality of life. Eur Spine J Off Publ Eur Spine Soc Eur Spinal Deform Soc Eur Sect Cerv Spine Res Soc 22(9):2015-2021

39. Roder C, Boszczyk B, Perler G, Aghayev E, Kulling F, Maestretti $\mathrm{G}$ (2013) Cement volume is the most important modifiable predictor for pain relief in BKP: results from SWISSspine, a nationwide registry. Eur Spine J Off Publ Eur Spine Soc Eur Spinal Deform Soc Eur Sect Cerv Spine Res Soc 22(10):2241-2248

40. He D, Wu L, Sheng X, Xiao Q, Zhu Y, Yu W et al (2013) Internal fixation with percutaneous kyphoplasty compared with simple percutaneous kyphoplasty for thoracolumbar burst fractures in elderly patients: a prospective randomized controlled trial. Eur Spine J Off Publ Eur Spine Soc Eur Spinal Deform Soc Eur Sect Cerv Spine Res Soc 22(10):2256-2263

41. Heintel TM, Berglehner A, Meffert R (2013) Accuracy of percutaneous pedicle screws for thoracic and lumbar spine fractures: a prospective trial. Eur Spine J Off Publ Eur Spine Soc Eur Spinal Deform Soc Eur Sect Cerv Spine Res Soc 22(3):495-502

42. Kashii M, Yamazaki R, Yamashita T, Okuda S, Fujimori T, Nagamoto $\mathrm{Y}$ et al (2013) Surgical treatment for osteoporotic vertebral collapse with neurological deficits: retrospective comparative study of three procedures - anterior surgery versus posterior spinal shorting osteotomy versus posterior spinal fusion using vertebroplasty. Eur Spine J Off Publ Eur Spine Soc Eur Spinal Deform Soc Eur Sect Cerv Spine Res Soc 22(7):1633-1642

43. Gakhar H, Swamy GN, Bommireddy R, Calthorpe D, Klezl Z (2013) A study investigating the validity of modified Tokuhashi score to decide surgical intervention in patients with metastatic spinal cancer. Eur Spine J Off Publ Eur Spine Soc Eur Spinal Deform Soc Eur Sect Cerv Spine Res Soc 22(3):565-568

44. Quraishi NA, Rajagopal TS, Manoharan SR, Elsayed S, Edwards KL, Boszczyk BM (2013) Effect of timing of surgery on neurological outcome and survival in metastatic spinal cord compression. Eur Spine J Off Publ Eur Spine Soc Eur Spinal Deform Soc Eur Sect Cerv Spine Res Soc 22(6):1383-1388

45. Skold C, Tropp H, Berg S (2013) Five-year follow-up of total disc replacement compared to fusion: a randomized controlled trial. Eur Spine J Off Publ Eur Spine Soc Eur Spinal Deform Soc Eur Sect Cerv Spine Res Soc 22(10):2288-2295

46. Rohlmann A, Lauterborn S, Dreischarf M, Schmidt H, Putzier M, Strube P et al (2013) Parameters influencing the outcome after total disc replacement at the lumbosacral junction. Part 1: misalignment of the vertebrae adjacent to a total disc replacement affects the facet joint and facet capsule forces in a probabilistic finite element analysis. Eur Spine J Off Publ Eur Spine Soc Eur Spinal Deform Soc Eur Sect Cerv Spine Res Soc 22(10):2271-2278

47. Strube P, Hoff EK, Schmidt H, Dreischarf M, Rohlmann A, Putzier M (2013) Parameters influencing the outcome after total disc replacement at the lumbosacral junction. Part 2: distraction and posterior translation lead to clinical failure after a mean follow-up of 5 years. Eur Spine J Off Publ Eur Spine Soc Eur 
Spinal Deform Soc Eur Sect Cerv Spine Res Soc 22(10):2279-2287

48. Duncan JW, Bailey RA (2013) An analysis of fusion cage migration in unilateral and bilateral fixation with transforaminal lumbar interbody fusion. Eur Spine J Off Publ Eur Spine Soc Eur Spinal Deform Soc Eur Sect Cerv Spine Res Soc 22(2):439-445

49. Kibsgard TJ, Roise O, Sudmann E, Stuge B (2013) Pelvic joint fusions in patients with chronic pelvic girdle pain: a 23-year follow-up. Eur Spine J Off Publ Eur Spine Soc Eur Spinal Deform Soc Eur Sect Cerv Spine Res Soc 22(4):871-877

50. Slinkard N, Agel J, Swiontkowski MF (2013) Documentation of outcomes for sacroiliac joint fusion: does prior spinal fusion influence the outcome? Eur Spine J Off Publ Eur Spine Soc Eur Spinal Deform Soc Eur Sect Cerv Spine Res Soc 22(10): 2318-2324

51. Havakeshian S, Mannion AF (2013) Negative beliefs and psychological disturbance in spine surgery patients: a cause or consequence of a poor treatment outcome? Eur Spine J Off Publ Eur Spine Soc Eur Spinal Deform Soc Eur Sect Cerv Spine Res Soc 22(12):2827-2835

52. Andersen T, Bunger C, Sogaard R (2013) Long-term health care utilisation and costs after spinal fusion in elderly patients. Eur Spine J Off Publ Eur Spine Soc Eur Spinal Deform Soc Eur Sect Cerv Spine Res Soc 22(5):977-984

53. Harroud A, Labelle H, Joncas J, Mac-Thiong JM (2013) Global sagittal alignment and health-related quality of life in lumbosacral spondylolisthesis. Eur Spine J Off Publ Eur Spine Soc Eur Spinal Deform Soc Eur Sect Cerv Spine Res Soc 22(4):849-856

54. Zhu B, Xu Y, Liu X, Liu Z, Dang G (2013) Anterior approach versus posterior approach for the treatment of multilevel cervical spondylotic myelopathy: a systemic review and meta-analysis. Eur Spine J Off Publ Eur Spine Soc Eur Spinal Deform Soc Eur Sect Cerv Spine Res Soc 22(7):1583-1593

55. Yoshida G, Kanemura T, Ishikawa $Y$, Matsumoto A, Ito Z, Tauchi R et al (2013) The effects of surgery on locomotion in elderly patients with cervical spondylotic myelopathy. Eur Spine J Off Publ Eur Spine Soc Eur Spinal Deform Soc Eur Sect Cerv Spine Res Soc 22:2545-2551

56. Malone A, Meldrum D, Gleeson J, Bolger C (2013) Electromyographic characteristics of gait impairment in cervical spondylotic myelopathy. Eur Spine J Off Publ Eur Spine Soc Eur Spinal Deform Soc Eur Sect Cerv Spine Res Soc 22:2538-2544

57. Obernauer J, Galiano K, Gruber H, Bale R, Obwegeser AA, Schatzer R et al (2013) Ultrasound-guided versus computed tomography-controlled periradicular injections in the middle and lower cervical spine: a prospective randomized clinical trial. Eur Spine J Off Publ Eur Spine Soc Eur Spinal Deform Soc Eur Sect Cerv Spine Res Soc 22:2532-2537

58. McCracken B, Klineberg E, Pickard B, Wisner DH (2013) Flexion and extension radiographic evaluation for the clearance of potential cervical spine injures in trauma patients. Eur Spine J Off Publ Eur Spine Soc Eur Spinal Deform Soc Eur Sect Cerv Spine Res Soc 22(7):1467-1473

59. Gumina S, Carbone S, Albino P, Gurzi M, Postacchini F (2013) Arm squeeze test: a new clinical test to distinguish neck from shoulder pain. Eur Spine J Off Publ Eur Spine Soc Eur Spinal Deform Soc Eur Sect Cerv Spine Res Soc 22(7):1558-1563

60. Fengbin Y, Xinwei W, Haisong Y, Yu C, Xiaowei L, Deyu C (2013) Dysphagia after anterior cervical discectomy and fusion: a prospective study comparing two anterior surgical approaches. Eur Spine J Off Publ Eur Spine Soc Eur Spinal Deform Soc Eur Sect Cerv Spine Res Soc 22(5):1147-1151

61. Park JY, Kim KH, Kuh SU, Chin DK, Kim KS, Cho YE (2013) What are the associative factors of adjacent segment degeneration after anterior cervical spine surgery? Comparative study between anterior cervical fusion and arthroplasty with 5-year follow-up MRI and CT. Eur Spine J Off Publ Eur Spine Soc Eur Spinal Deform Soc Eur Sect Cerv Spine Res Soc 22(5):1078-1089

62. Millet JP, Moreno A, Fina L, del Bano L, Orcau A, de Olalla PG et al (2013) Factors that influence current tuberculosis epidemiology. Eur Spine J Off Publ Eur Spine Soc Eur Spinal Deform Soc Eur Sect Cerv Spine Res Soc 22(Suppl 4):539-548

63. Rajasekaran S, Khandelwal G (2013) Drug therapy in spinal tuberculosis. Eur Spine J Off Publ Eur Spine Soc Eur Spinal Deform Soc Eur Sect Cerv Spine Res Soc 22(Suppl 4):587-593

64. (1978) Five-year assessments of controlled trials of ambulatory treatment, debridement and anterior spinal fusion in the management of tuberculosis of the spine. Studies in Bulawayo (Rhodesia) and in Hong Kong. Sixth report of the Medical Research Council Working Party on tuberculosis of the spine. J Bone Jt Surg Br Vol 60-b(2):163-177

65. Cheung WY, Luk KD (2013) Clinical and radiological outcomes after conservative treatment of TB spondylitis: is the 15 years' follow-up in the MRC study long enough? Eur Spine J Off Publ Eur Spine Soc Eur Spinal Deform Soc Eur Sect Cerv Spine Res Soc 22(Suppl 4):594-602

66. Rajasekaran S, Vijay K, Shetty AP (2010) Single-stage closingopening wedge osteotomy of spine to correct severe post-tubercular kyphotic deformities of the spine: a 3-year follow-up of 17 patients. Eur Spine J Off Publ Eur Spine Soc Eur Spinal Deform Soc Eur Sect Cerv Spine Res Soc 19(4):583-592

67. Jain AK, Kumar J (2013) Tuberculosis of spine: neurological deficit. Eur Spine J Off Publ Eur Spine Soc Eur Spinal Deform Soc Eur Sect Cerv Spine Res Soc 22(Suppl 4):624-633

68. Crostelli M, Mazza O, Mariani M, Mascello D (2013) Treatment of severe scoliosis with posterior-only approach arthrodesis and all-pedicle screw instrumentation. Eur Spine J 22(Suppl 6):S808S814 